\title{
Effects of a manualized short-term treatment of internet and computer game addiction (STICA): study protocol for a randomized controlled trial
}

\author{
Susanne Jäger ${ }^{1 *}$, Kai W Müller ${ }^{1}$, Christian Ruckes ${ }^{2}$, Tobias Wittig ${ }^{2}$, Anil Batra ${ }^{3}$, Michael Musalek $^{4}$, Karl Mann $^{5}$,
} Klaus Wölfling ${ }^{1}$ and Manfred E. Beutel ${ }^{1}$

\begin{abstract}
Background: In the last few years, excessive internet use and computer gaming have increased dramatically. Salience, mood modification, tolerance, withdrawal symptoms, conflict, and relapse have been defined as diagnostic criteria for internet addiction (IA) and computer addiction (CA) in the scientific community. Despite a growing number of individuals seeking help, there are no specific treatments of established efficacy.

Methods/design: This clinical trial aims to determine the effect of the disorder-specific manualized short-term treatment of IA/CA (STICA). The cognitive behavioural treatment combines individual and group interventions with a total duration of 4 months. Patients will be randomly assigned to STICA treatment or to a wait list control group. Reliable and valid measures of IA/CA and co-morbid mental symptoms (for example social anxiety, depression) will be assessed prior to the beginning, in the middle, at the end, and 6 months after completion of treatment.
\end{abstract}

Discussion: A treatment of IA/CA will establish efficacy and is desperately needed. As this is the first trial to determine efficacy of a disorder specific treatment, a wait list control group will be implemented. Pros and cons of the design were discussed.

Trial Registration: ClinicalTrials (NCT01434589)

Keywords: Internet addiction, computer game addiction, STICA, intervention, cognitive behavioral therapy

\section{Background}

The internet has become accessible for the great majority of the population (for example flat rates, WLAN, or portable computers). In a representative German sample $(\mathrm{n}=2475)$ in 2009 the rate of leisure-time internet users for women was about $51 \%$ and for men about $60 \%$. The most frequently used internet applications were email (93\%), information and research (92\%), shopping (76\%), and chatting (62\%) [1]. In 2004 about 68\% of American adults used the internet regularly and $4 \%$ to $14 \%$ showed one or more markers of problematic use with a prevalence of internet addiction (IA) at about 1\% [2], which is concordant with an actual German study [3]. The onset of manifest addicted behavior is reported in the late 20s or

\footnotetext{
* Correspondence: Susanne.jaeger@unimedizin-mainz.de

'Outpatient Clinic for Behavioural Addictions, Department of Psychosomatic Medicine and Psychotherapy, University Medical Center of the Johannes

Gutenberg University Mainz, Mainz, Germany

Full list of author information is available at the end of the article
}

early 30 s age groups [2]. In epidemiological studies, prevalence rates of addicted internet use and computer game behavior range between $1.5 \%$ to $3.0 \%$ in German $[3,4]$ and Austrian [5] adolescents, respectively.

According to Block [6], three subtypes of IA/computer game addiction (CA) (excessive gaming, sexual preoccupations, and email/text messaging) have four components in common: (a) excessive use (along with a loss of sense of time or the ignorance of basic drives); (b) withdrawal (for example tension, anger, agitation, and/or depression when access to a computer is blocked; (c) tolerance (increasing use or sophistication of computer equipment); and (d) negative repercussions (for example poor achievement/ performance, fatigue, social isolation, or conflicts).

Salience, mood modification, tolerance, withdrawal symptoms, conflict, and relapse are additional diagnostic criteria for IA and CA [7]. The addicted individual is increasingly attracted towards the excessive behavior and 
life is emotionally and cognitively preoccupied with the application (for example computer game), requiring more and more time in order to regulate his mood states. Empirical studies $[4,8,9]$ have demonstrated that the symptom complex of IA/CA $[10,11]$ matches the criteria of substance disorders. Results of neurobiological studies have identified neurophysiological mechanisms in IA/CA equivalent to substance abuse (alcohol [12] and cannabis addiction [13]).

Patients with CA and IA have increasingly sought help in addiction counseling [14], because of serious negative psychosocial consequences (social, work/education, health) which have been documented along with high mental comorbidities [15-19]. IA is strongly associated with dimensionally measured depression [18,20], indicators of social isolation or behavioral deficits (for example ADHD $[18,21,22])$, or impulsivity [23]. In the Grüsser-Sinopoli outpatient clinic for behavioral addiction, from 2008 to 2010, a total of 326 patients have been assessed for IA/CA by clinical examination and tests. Of those, 192 patients were classified as IA/CA. They were predominantly (97\%) male and aged from 18 to 30 years. They showed strong evidence of social phobia and depression as well as performance decrements in school and work.

Despite its increasing importance as a major health problem among adolescents and young adults at present, there is still a lack of evidence-based interventions for IA/CA. Preliminary evidence has only been generated in open trials for non-European and Asian populations [24,25].

Therefore, a specific short-term treatment program for IA/CA, based on cognitive behavior therapy (STICA) was developed. A preliminary evaluation of the manualized STICA treatment was performed in an open trial of the Grüsser-Sinopoli outpatient clinic for behavioral addiction with a total of 33 patients. Twenty-four out of this sample completed STICA regularly, nine patients terminated treatment prematurely and were considered as drop-outs (27\%). Based on the full sample of 33 patients (intent to treat analysis) criteria for treatment response (primary efficacy endpoint) were reached by $67 \%$ which corresponds to a large effect size of 1.27 [Wölfling K, Müller KW, Beutel ME: Treatment outcome of a manualized cognitive behavior therapy in Internet and Computer game addiction, unpublished].

This study will assess the efficacy of the manualized STICA. Furthermore, the durability of treatment response in these patients and the impact on associated psychiatric symptoms (for example social anxiety and depression) will be determined. Currently STICA is the only manualized outpatient treatment program for IA/ CA in Germany [26] and further international concepts and clinical trials were not methodologically convincing [27].

\section{Methods/Design}

\section{Study centers}

This multicenter study is coordinated by the outpatient clinic for behavioral addictions of the Clinic for Psychosomatic Medicine and Psychotherapy of the University Medical Center Mainz. Three centers will further participate, the Anton-Proksch-Institute, Austria, the Section Addiction Medicine and Addiction Research of the University Hospital Tübingen, and the Addiction Medicine of the Central Institute of Mental Health in Mannheim.

Investigators in all centers are psychotherapists (physicians or psychologists) and experts in the treatment of addiction behavior.

\section{Participants}

Patients will be included, if the following eight inclusion criteria are fulfilled: (1) IA/CA according to the AICA (Assessment of Internet and Computer game Addiction) expert rating for at least 6 months and (2) a score $\geq 7$ in the AICA self-report IA/CA. (3) Patients with co-morbid disorders will be included, provided that IA/CA is the primary diagnosis. The study will only include (4) men in (5) the age between 17 and 45 years. (6) If the patients are currently on psychotropic medications, no changes in medications and dosages in the past 2 months and during STICA treatment are allowed. (7) If currently off all psychotropic medications, patient must have been off at least 4 weeks. (8) During STICA no other ongoing psychotherapy is allowed and previous psychotherapy must have been completed for at least 4 weeks.

Patients with a score $<40$ in the Global Assessment of Functioning (GAF [28]) or severe major depression (Beck Depression Inventory; BDI-II [29] $\geq 29$ ) are excluded. Additional exclusion criteria are current alcohol or drug addictions, borderline, antisocial, schizoid, and schizotypal personality disorders, a lifetime diagnosis of schizophrenia, schizoaffective, bipolar, or organic mental disorder and a current unstable medical illness.

Over a time period of 36 months we plan to include 192 patients in the study. The patients will be randomly assigned to the intervention or to the wait list control group (WLC). Prior to randomization, a total of 18 patients have to be allocated to the trial. The intervention group will start treatment immediately after randomization, whereas the WLC group has to wait over a period of 4 months, until they will receive the same therapy.

\section{Intervention}

The manualized STICA [26] is based on a cognitive behavior approach and combines group with individual therapy. STICA comprises 23 psychotherapy sessions with a total duration of 4 months. Fifteen out of twentythree sessions will be weekly group sessions (100 min 
each) and eight will be fortnightly individual sessions (50 min).

Table 1 shows treatment phases and strategies during the early, middle, and termination phases. Based on understanding the mechanisms and the consequences of IA/CA (early phase), patients are trained to identify the triggers of their own dysfunctional internet use. By using diaries, social skill training, and exposition training, patients learn to reduce and to control their computer and internet use. In the termination phase of treatment, tools will be transferred to daily life and strategies for relapse prevention will be discussed.

\section{Assessment}

Figure 1 shows a flow chart of the five time points of assessment. At T0a patients are informed about the study and assessed for eligibility. Patients fill out the AICA-S [30,31, Müller KW, Glaesmer H, Brähler E, Wölfling $\mathrm{K}$, Beutel $\mathrm{M}$; Internet addiction in the general population. Results from a german population-based

Table 1 STICA Treatment phases and strategies

\begin{tabular}{ll}
\hline Treatment phase & Key interventions \\
\hline Early phase & Patient education on mechanisms and effects \\
of IAVA (for example learning theories, \\
development and consequences of IA/CA, \\
vicious cycles of addiction, and so on) \\
Promotion of social communication and \\
bonding in the group and individual setting \\
Identification of the triggers of dysfunctional \\
internet use (for example emotional states, \\
maladaptive cognitions, daily hassles, and so \\
on) by keeping diaries \\
Functional analysis of the addictive behavior \\
Enabling functional internet use by appropriate \\
problem-solving strategies \\
Assisting the establishment of a social network \\
in real life \\
Building alternative activities \\
Self-monitoring to reduce procrastination \\
tendencies \\
Promotion of social communication \\
Exposition training (confronting and deleting \\
access to critical applications, for example the \\
self-created avatar) \\
Skill training (for example coping with stress \\
and problems, social skills, building alternative \\
activities, and so on) \\
Promotion of functional computer and internet \\
use prevention \\
Review of transfer of treatment tools to daily \\
life \\
Functional computer/internet use \\
Elaboration of tools preventing a relapse \\
\hline
\end{tabular}

survey. unpublished] and the BDI-II [29]. AICA-S values range between 0 and 27 , and scores $\geq 7$ have been defined as problematic internet use. Therapists assess onset, course, and criteria of IA/CA, treatment history, motivation for therapy, and the GAF [28]. The AICA Checklist will be rated by an independent and blinded rater.

The assessment T0b is performed immediately before randomization and commencement of treatment. The criteria for IA/CA will be rechecked by self-report measurements, if the delay due to the group recruitment exceeds 2 weeks. Therapists fill out the GAF [28] and collect information about medications, other therapies, and treatment history. An independent and blinded rater will assess mental disorders with the SCID-I/II [32] and conduct the AICA-Checklist. A drug screening is further used to assess objective information on drug consumption. Self-report assessment includes IA/CA (AICA-S [30,31]), depression (BDI-II [29]), obsessive-compulsive behavior (SCL-90-R [33]), generalized anxiety and panic (Patient Health Questionnaire [34]), somatization [34], general distress [34], depersonalization (CDS-2 [35]), and social fear (LSAS [36]). Patients also fill out dimensions of personality (NEO-FFI [37]), attention deficit disorder (WURS-k [38]), self-efficacy (SWE [39,40]), positive and negative affectivity (PANAS [41]), and adverse childhood experiences (ACE [42]). Finally, they answer questions concerning perceived stress (PSS [43]) and about their life satisfaction (FLZ [44]). Attention of the patients will be checked with the $\mathrm{d} 2$ [45].

After 2 months of therapy (T1) patient-rated outcome measures are reapplied (AICA, GAF, BDI-II) and drug screening repeated. The outcome measures are supplemented by assessments of group climate (GCQ [46]) and therapeutic alliance (HAQ [47]).

Immediately after completion of the intervention (T2) patients fill out a set of questionnaires identical with the set at TOb, except for the trait measures (NEO-FFI, WURS-k, ACE). Group climate and therapeutic alliance are assessed additionally. Drug screening is applied and is obligatory. For patients of the WLC group this is the final assessment. Shortly after the survey their intervention will start.

Patients of the intervention group are asked to evaluate the stability of treatment effects 6 months after termination of treatment (T3). Thereby the used set of questions corresponds to $\mathrm{T} 2$.

\section{Data collection}

In this study there are two sources of electronic study data. An eCRF has been developed for the investigators to document their study data in a database stored, maintained, and administered by the IZKS Mainz. It is 


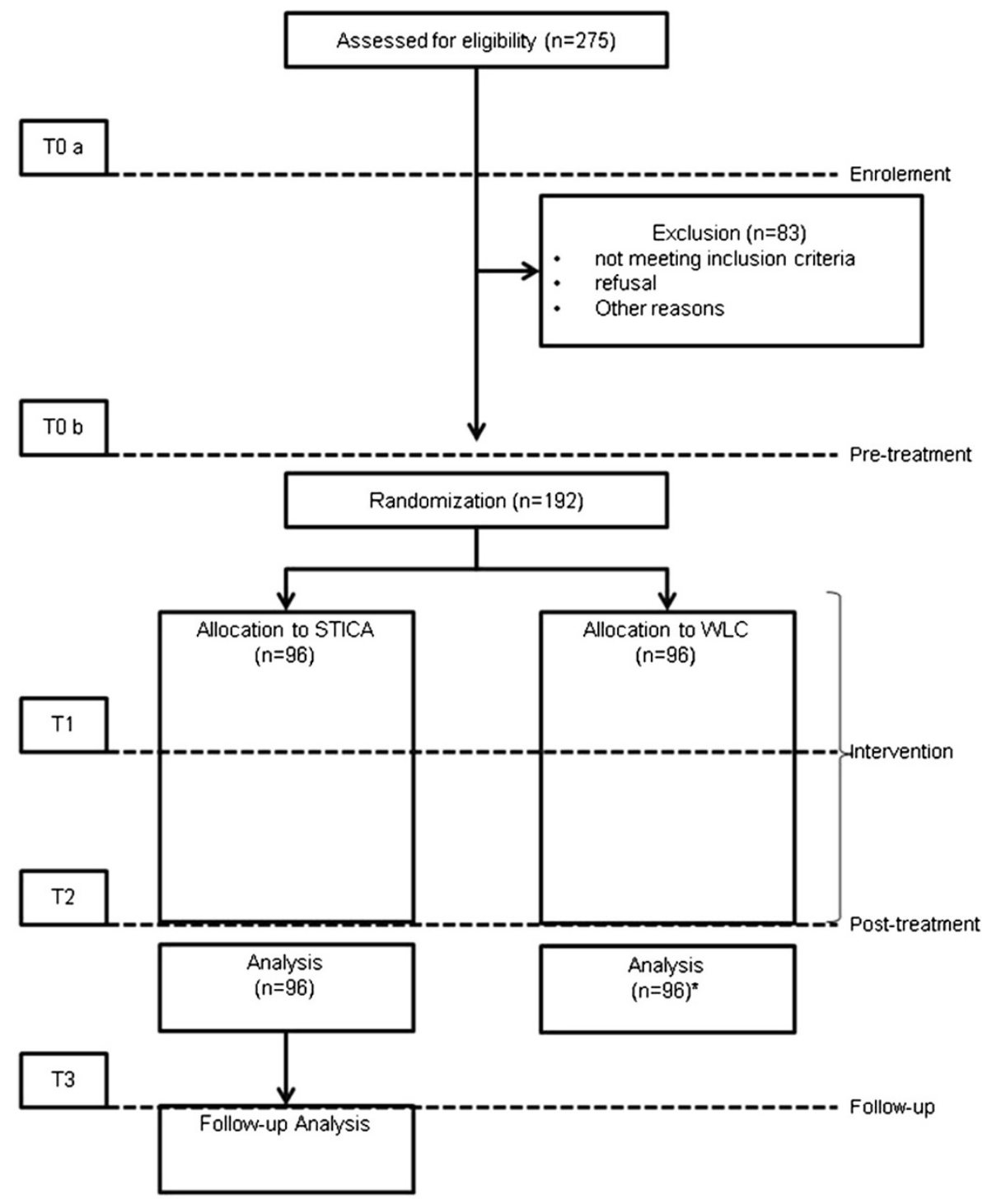

Figure 1 Flow chart of the study. Patients of the wait list control group (WLC) will be offered STICA treatment after the intervention group has finished. The follow-up analysis will be performed separately for WLC.

password protected with individual accounts for all investigators.

Patients will answer the self-report questionnaires by entry forms customized for iPADs. Each patient receives only access to his own current questionnaire.

After data collection, eCRF and iPAD data will be transformed into one SAS database for evaluation.

\section{Objectives and hypotheses}

The purposes or this study are to determine the efficacy of STICA, to assess the durability of treatment response in these patients, and the impact on associated mental symptoms (for example social anxiety and depression).

\section{Outcomes}

The primary efficacy endpoint is defined as improvement of IA/CA rated by the patient himself (primary outcome measure: AICA-S [30,31]). At the end of therapy an AICA-S score $<7$ indicates remission.

Secondary endpoints include the remission of IA/CA in the expert rating (AICA-C $\leq 13$ ). The preoccupation with the internet or computer games will be analyzed (hours spent per week). IA and CA are associated with negative consequences in health, social communication, psychosocial wellbeing (GAF [28], BDI-II [30], LSAS [36]), level of performance in school or work, and self-efficacy (SWE [39]). For each instrument assessments at baseline will be compared to assessments obtained 4 and 6 months after therapy. 


\section{Sample size calculation}

The sample size calculation is based on the primary endpoint (T2: end of therapy) and a chi-square test without continuity correction on a two-sided level of significance of 0.05 . The calculation is based on results from 33 patients, who participated in an open trial. Twenty-four patients improved according to the AICA-S $<7$. A difference to the control group of $20 \%$ is considered as clinically relevant. With a power of $90 \%, 184$ patients in total are needed to detect that difference. Considering the average therapy group size of eight, 16 subjects have to be randomized at the same time. Therefore, we will need to include 192 patients in this trial $(n=96$ patients for each group). The primary analysis will be performed on the population of all randomized subjects (intention to treat (ITT) population). Subjects who discontinue the therapy will be regarded as non-improvers to treatment. Our previous experience with internet addicts revealed drop-out rates of about 27\% (nine drop-outs from 33 patients).

\section{Randomization}

Patients will be randomly assigned either to the STICA intervention group or to the WLC group. The randomization list will be generated stratified by the Interdisciplinary Centre for Clinical Trials (IZKS). Considering the average therapy group size of eight patients, 16 patients have to be randomized at the same time. The randomization ratio will be 1:1 within each center. After confirmation that a patient fulfils all inclusion criteria for randomization, the electronic case report form (eCRF) will immediately provide the investigator with the randomization results. Patients are subsequently informed about the randomization result and the intervention starts shortly after randomization. The IZKS will furthermore insure treatment integrity by regular site visits.

\section{Statistical analysis Primary analysis}

The primary efficacy endpoint is defined as the change of the AICA-S level. This will be analyzed using a logistic regression model with predictors of group (STICA treatment $v s$. WLC), pre-treatment score of AICA-S, education, trial center, and age.

The primary hypothesis to be tested is:

$$
\mathrm{H}_{0}: \pi_{\mathrm{STICA}}=\pi_{\mathrm{WLC}} v s . \mathrm{H}_{1}: \pi_{\mathrm{STICA}} \neq \pi_{\mathrm{WLC}}
$$

where $\pi_{\mathrm{STICA}}$ and $\pi_{\mathrm{WLC}}$ are the probabilities to respond to treatment in the STICA treatment group and the WLC group, respectively. The primary analysis will be performed on the ITT population on a two-sided level of significance $\alpha=0.05$. The two-sided level of significance will be the same for all analyses. A completer analysis will be performed for sensitivity. Additionally, the analysis will be repeated with a predictor for the therapy group. Drop-outs during the treatment phase will be regarded as treatment failures.

\section{Secondary analysis}

The remission of IA/CA according to the AICA Checklist will be examined using logistic regression analyses with the same predictors as the primary analysis. The reduction of negative consequences, GAF, depression (BDI-II), and social anxiety (LSAS) will be examined using ANCOVA with covariates.

Analyses will be conducted on a two-sided level of significance of $\alpha=0.05$. Descriptive statistics are used to show changes over time. Serious adverse events and drop-outs will be analyzed by using descriptive statistics.

\section{Safety aspects}

Safety parameters will comprise newly occurring psychiatric diagnoses (SCID-I [32]) and all serious adverse events that are reported during and up to 6 months after treatment. Therefore in the context of psychotherapy suicidal ideations or the global functioning level will be regarded.

\section{Medical complications}

According to GCP, an adverse event (AE) is defined as follows: any untoward medical occurrence in a patient participating in a clinical trial. An $\mathrm{AE}$ can therefore be any unfavorable and unintended sign (including an abnormal laboratory finding), symptom, or disease, whether or not related to the trial intervention. Due to the fact that this trial analyses a psychological treatment, only AEs concerning psychological conditions, defined as any disorder classified by the International Classification of Diseases [48] F00-F99 ('Mental and Behavioral Disorders') will be documented.

For this study the following conditions were defined as AE: (1) new symptoms/medical conditions, (2) new diagnosis, (3) intercurrent diseases and accidents, (4) worsening of medical conditions/diseases existing before clinical trial start, (5) recurrence of disease, or (6) increase of frequency or intensity of episodical diseases.

A serious adverse event (SAE) is an AE that: (1) results in death, (2) is life-threatening, (3) requires patient hospitalization or prolongation of existing hospitalization, (4) results in persistent or significant disability/incapacity, or (5) is a congenital anomaly/birth defect.

All medical complications during the study are documented in the eCRF. 


\section{Ethical issues}

Clinical protocol and written informed consent were approved by the Ethics Committee (EC) of the Federal State of Rhineland Palatinate (Germany), which is responsible for the coordinating centre Mainz (Ref. No. 837.316.11 (7858)). Ethics Committees of all cooperating centers will provide the necessary additional documents.

All procedures described in the clinical trial protocol follow the ICH-GCP guidelines and the ethical principles described in the current revision of the Declaration of Helsinki. The trial will be carried out in keeping with local legal and regulatory requirements.

Before being admitted to the clinical trial, patients receive detailed explanations of the nature, scope, and possible consequences of the clinical trial in a form understandable to them. The patients must give consent in writing. Each patient will receive a copy of the signed informed consent document.

In this clinical trial all patients, including the WLC group will receive the full treatment. For the WLC patients the therapy begins after a waiting period of 4 months.

An independent Data Monitoring and Safety Board (DMSB) has been established for this study. The DMSB will supervise the conduct of this trial and will issue recommendations for early termination, modifications or continuation of the trial, if necessary. The DMSB and the EC must be informed immediately of study-related SAE.

\section{Discussion}

The number of patients suffering from IA/CA who need professional help increases constantly. Up to now there is no specific manualized intervention program and there are no well-defined treatments of established efficacy. To our knowledge, STICA is the very first clinical trial for establishing efficacy of a specific treatment for IA/CA.

The efficacy of the treatment will be checked in a randomized controlled multicenter trial. The use of a WLC group appears to be justified because of the novel treatment approach and the lack of comparable approaches. Patients in the WLC are assured to receive full treatment after a waiting period of 4 months following randomization. Thus, however, follow-up of waitlist controls is not possible.

STICA will also regard co-morbid mental disorders and serious long-term consequences (for example social withdrawal or failure in school/education) caused by excessive internet or computer game use. The aim of STICA is the reintegration of the patients into a normal life, including controlled use of computer and internet, social contacts, and work performance.

The results of this study will be of high relevance because of the methodological demand and the high relevance of the topic. This study will determine the effectiveness and durability of a cognitive behavioral short-term treatment for IA/CA. For patient care it will be important to implement an effective treatment for IA/CA in clinical routine.

\section{Trial status}

The first patient was enrolled to the STICA study on February 1, 2012. Follow-up measures for the last included patients were expected to be terminated in June 2014.

\section{Abbreviations}

ACE: Adverse childhood experience questionnaire; AE: Adverse event; ADHD: Attention deficit hyperactivity disorder; AICA-S: Assessment of internet and computer game addiction, self report; AICA-Checklist: Assessment of internet and computer game addiction, expert rating; BDI-II: Beck Depression Inventory; CA: Computer game addiction; CDS-2: Cambridge depersonalization scale; DFG: Deutsche Forschungsgemeinschaft; DMSB: Data Monitoring and Safety Board; d2: Test of attention; EC: Ethics Committee; eCRF: Electronic Case Report Form; FLZ: Questionnaire of life satisfaction; GAF: Global Assessment of Functioning; GCP: Good Clinical Practice; HAQ: Helping alliance questionnaire; ICH: International Conference on Harmonisation of Technical Requirements for Registration of Pharmaceuticals for Human Use; IA: Internet addiction; ITT: Intention to treat;

IZKS: Interdisciplinary Centre for Clinical Trials; LSAS: Liebowitz social anxiety scale; NEO-FFI: NEO Five factor inventory; PANAS: Positive and negative affective schedule; PHQ: Patient health questionnaire; PSS: Perceived stress scale; SAE: Serious adverse event; SCID: I/II Structured clinical interview for DSM IV; SCL-90-R: Symptom Checklist 90 revised; STICA: Short-term Treatment of Internet and Computer game Addiction; SWE: Assessment of the expectance of self-efficacy; WLC: Wait list control; WURS-k: Wender Uta rating scale.

\section{Competing interests}

The authors declare that they have no competing interests.

\section{Acknowledgements}

The study is funded by the Deutsche Forschungsgemeinschaft (DFG) BE2248/ 10-1 and the German Federal Ministry of Education and Research (BMBF) and supported by the IZKS Mainz, which is founded by the BMBF (FKZ 01KN1103).

\section{Author details}

${ }^{1}$ Outpatient Clinic for Behavioural Addictions, Department of Psychosomatic Medicine and Psychotherapy, University Medical Center of the Johannes Gutenberg University Mainz, Mainz, Germany. ${ }^{2}$ Interdisciplinary Center for Clinical Trials (IZKS), University Medical Center of the Johannes Gutenberg University Mainz, Mainz, Germany. ${ }^{3}$ Section Addiction Medicine and Addiction Research, University Hospital Tübingen, Tübingen, Germany. ${ }^{4}$ Anton-Proksch Institute Wien, Wien, Austria. ${ }^{5}$ Addiction Medicine, Central Institute of Mental Health Mannheim, Mannheim, Germany.

\section{Authors' contributions}

SJ did the first draft of the manuscript and is contact person for questions about realization, design, and administration. SJ, MEB, and KW did the final draft of the manuscript and critically revised it for its intellectual content. KW and MEB developed the therapy, which will be evaluated with this study. The proposal was first prepared by KW, KWM, MEB, and CR. For the grant MEB and $\mathrm{KW}$ operate as principle and co-principle investigator. MEB is responsible for the proposal. KWM, CR, TW, KW, and MEB substantially contributed to the conception and the final design of the study. $A B, M M$, and $K M$ are

responsible for the correct realization of STICA in the different centers and cooperate to improve the study design. All authors read and approved the final manuscript.

Received: 3 January 2012 Accepted: 27 April 2012

Published: 27 April 2012 


\section{References}

1. Beutel ME, Brähler E, Glaesmer H, Kuss DJ, Wölfling K, Müller KW: Regular and problematic leisure-time Internet use in the community: results from a German population-based survey. Cyberpsychol Behav Soc Netw 2011, 14:291-296

2. Aboujaoude E, Koran L, Gamel N, Large M, Serpe R: Potential markers for problematic internet use: a telephone survey of 2,513 adults. CNS Spectrums 2006, 11:750-755.

3. Rumpf H-J, Meyer C, Kreuzer A, John U: Prävalenz der Internetabhängigkeit (PINTA). In Bericht an das Bundesministerium für Gesundheit: Universitäten Greifswald \& Lübeck; 2011.

4. Wölfling K, Thalemann R, Grüsser-Sinopoli SM: Computerspielsucht: Ein psychopathologischer Symptomkomplex im Jugendalter. Psychiatr Prax 2008, 35:226-232.

5. Batthyany D, Müller KW, Benker F, Wölfling K: Computerspielverhalten: Klinische Merkmale von Abhängigkeit und Missbrauch bei Jugendlichen. Wien Klin Wochenschr 2009, 121:502-509.

6. Block JJ: Issues for DSM-V: internet addiction. Am J Psychiatry 2008, 165:306-307.

7. Griffiths M: Does Internet and computer "Addiction" exist? Some case study evidence. Cyberpsychol Behav 2000, 3:211-218.

8. Rehbein F, Kleimann M, Mößle $\mathrm{T}$ : Computerspielabhängigkeit im Kindes- und Jugendalter - Empirische Befunde zu Ursachen, Diagnostik und Komorbiditäten unter besonderer Berücksichtigung spielimmanenter Abhängigkeitsmerkmale. In Kriminologisches Forschungsinstitut Niedersachsen eV Forschungsbericht Nr 108; 2009.

9. Morrison CM, Gore $\mathrm{H}$ : The relationship between the excessive Internet use and depression: A questionnaire-based study of 1,319 young people and adults. Psychopathology 2010, 43:121-126.

10. Yen JY, Ko CH, Yen CF, Chen SH, Chung WL, Chen CC: Psychiatric symptoms in adolescents with Internet addiction: Comparison with substance use. Psychiatry Clin Neurosci 2008, 62:9-16.

11. Thalemann R, Wolfling K, Grusser SM: Specific cue reactivity on computer game-related cues in excessive gamers. Behav Neurosci 2007, 121:614-618.

12. Hermann MJ, Weijers H-G, Wiesbeck GA, Böning J, Fallgatter AJ: Alcohol cue-reacitivity in heavy and light social drinkers as revealed by eventrelated potentials. Alcohol 2001, 36:588-593.

13. Wölfling K, Flor H, Grüsser SM: Psychophysiological responses to drugassociated stimuli in chronic heavy cannabis use. Eur J Neurosci 2008, 27:976-983.

14. Wessel T, Müller KW, Wölfling K: Computerspielsucht: Erste Fallzahlen aus der Suchtkrankenhilfe. In DHS Jahrbuch Sucht. Edited by Deutsche Hauptstelle für Suchtfragen e.V. (DHS). Geesthacht: Neuland; 2009

15. Beutel ME, Hoch C, Wölfling K, Müller KW: Klinische Merkmale der Computerspiel- und Internetsucht am Beispiel der Inanspruchnehmer einer Spielsuchtambulanz. Z Psychosom Med Psychother 2011, 57:77-90.

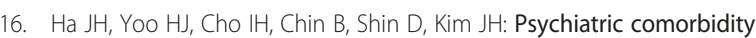
assessed in Korean children and adolescents who screen positive for Internet addiction. J Clin Psychiatry 2006, 67:821-826

17. Peukert P, Sieslack S, Barth G, Batra A: Internet- und Computerspielabhängigkeit: Phänomenologie, Komorbiditat, Ätiologie, Diagnostik und therapeutische Implikationen fur Betroffene und Angehörige. Psychiatr Prax 2010, 37:219-224.

18. Yen JY, Ko CH, Yen CF, Wu HY, Yang MJ: The comorbid psychiatric symptoms of Internet addiction: attention deficit and hyperactivity disorder (ADHD), depression, social phobia, and hostility. J Adolesc Health 2007, 41:93-98.

19. Bernardi S, Pallanti S: Internet addiction: a descriptive clinical study focusing on comorbidities and dissociative symptoms. Compr Psychiatry 2009, 50:510-516.

20. Kim K, Ryu E, Chon MY, Yeun EJ, Choi SY, Seo JS, Nam BW: Internet addiction in Korean adolescents and its relation to depression and suicidal ideation: a questionnaire survey. Int J Nurs Stud 2006, 43:185-192

21. Yoo HJ, Cho SC, Ha J, Yune SK, Kim SJ, Hwang J, Chung A, Sung YH, Lyoo IK: Attention deficit hyperactivity symptoms and internet addiction. Psychiatry Clin Neurosci 2004, 58:487-494.
22. Yen JY, Yen CF, Chen CS, Tang TC, Ko CH: The association between adult $\mathrm{ADHD}$ symptoms and internet addiction among college students: the gender difference. Cyberpsychol Behav 2009, 12:187-191.

23. Cao F, Su L, Liu T, Gao X: The relationship between impulsivity and Internet addiction in a sample of Chinese adolescents. Eur Psychiatry 2007, 22:466-471.

24. Du YS, Jiang W, Vance A: Longer term effect of randomized, controlled group cognitive behavioural therapy for Internet addiction in adolescent students in Shanghai. Aust N Z J Psychiatry 2010, 44:129-134

25. Young KS: Cognitive behavior therapy with Internet addicts: treatment outcomes and implications. Cyberpsychol Behav 2007, 10:671-679.

26. Wölfling K, Jo C, Bengesser I, Beutel ME, Müller KW: Computerspiel- und Internetsucht. Ein kognitiv behaviorales Behandlungsmanual. Stuttgart: Kohlhammer; in prep.

27. King DL, Delfabbro $\mathrm{PH}$, Griffiths MD, Gradisar M: Assessing clinical trials of Internet addiction treatment: A systematic review and CONSORT evaluation. Clin Psychol Rev 2011, 31:1110-1116.

28. Saß H: Wittchen H-U, Zaudig M, Houben I: Diagnostische Kriterien DSM-IV Göttingen: Hogrefe; 1998

29. Hautzinger M, Keller F, Kühner C: Beck Depressions Inventar: Revision (BDI-II). Frankfurt a.M.: Harcourt Test Services; 2006.

30. Wölfling K, Müller KW, Beutel M: Reliabilität und Validität der Skala zum Computerspielverhalten (CSV-S). Psychother Psychosom Med Psychol 2011, 61:216-224.

31. Wölfling K, Müller KW: Pathologisches Glücksspiel und Computerspielabhängigkeit. Wissenschaftlicher Kenntnisstand zu zwei Varianten substanzungebundener Abhängigkeitserkrankungen. Bundesgesundheitsblatt Gesundheitsforschung Gesundheitsschutz 2010, 53:306-312.

32. Wittchen H-U, Zaudig M, Fydrich T: Strukturiertes Klinisches Interview für DSMN. Göttingen: Hogrefe; 1997.

33. Franke GH: Symptom-Checkliste von L.R. Derogatis (SCL-90-R) - deutsche Version. 2nd edition. Göttingen: Beltz Test; 2002.

34. Löwe B, Spitzer RL, Zipfel S, Herzog W: PHQ-D: Gesundheitsfragebogen für Patienten - Kurzanleitung zur Komplettversion und Kurzform. Heidelberg: Medizinische Universitätsklinik Heidelberg; 2002.

35. Michal M, Zwerenz R, Tschan R, Edinger J, Lichy M, Knebel A, Tuin I, Beutel M: Screening nach Depersonalisation-Derealisation mittels zweier Items der Cambridge Depersonalisation Scale. Psychother Psych Med 2010, 60:175-179.

36. Stangier U, Heidenreich T: Die Liebowitz Soziale Angst -Skala (LSAS). In Skalen für Psychiatrie. Edited by Collegium Internationale Psychiatriae Scalarum. Göttingen: Beltz Test; 2004.

37. Borkenau P, Ostendorf F: NEO-Fünf-Faktoren-Inventar nach Costa und McCrae (NEO-FFI). 2nd edition. Göttingen: Hogrefe; 2008.

38. Retz-Junginger $P$, Retz W, Blocher D, Weijers H-G, Trott G-E, Wender PH, Rössler M: Wender Utah Rating Scale (WURS-k): Die deutsche Kurzform zur retrospektiven Erfassung des hyperkinetischen Syndroms bei Erwachsenen. Der Nervenarzt 2002, 73:830-838.

39. Schwarzer R, Jerusalem M: Skalen zur Erfassung von Lehrer- und schülermerkmalen. Dokumentation der psychometrischen Verfahren im rahmen der wissenschaftlichen Begleitung des Modellversuchs Selbstwirksame Schulen. Berlin: Freie Universität Berlin; 1999.

40. Schwarzer R, Mueller J, Greenglass E: Assessment of perceived general selfefficacy on the internet: Data collection in cyberspace. Anxiety Stress Coping 1999, 12:145-161.

41. Krohne HW, Egloff B, Kohlmann C-W, Tausch A: Untersuchung mit einer deutschen Form der Positive and Negative Affect Schedule (PANAS). Diagnostica 1996, 42:139-156.

42. Schäfer I, Spitzer C: Deutsche Version des "Adverse Childhood Experiences Questionnaire (ACE)". Hamburg: Universität Hamburg; 2009.

43. Cole S: Assessment of differential item functioning in the Perceived Stress Scale-10. J Epidemiol Community Health 1999, 53:319-320

44. Heinrich G, Herschbach P: Questions of Life Satisfaction (FLZM) - A short questionnaire for assessing subjective quality of life. Eur J Psychol Assess 2000, 16:150-159.

45. Brickenkamp R: Test $d 2$ - Aufmerksamkeits-Belastungs-Test. 9th edition. Göttingen: Hogrefe; 2002. 
46. Mackenzie RK, Tschuschke V: Relatedness, group work, and outcome in long-term inpatient psychotherapy groups. J Psychotherpay Pract Res 1993, 2:147-156.

47. Bassler M, Potratz B, Krauthauser H: Der "Helping Alliance Questionnaire" (HAQ) von Luborsky. Psychotherapeut 1995, 40:23-32.

48. Dilling H: Taschenführer zur ICD-10-Klassifikation psychischer Störungen. Bern: Huber; 2010.

doi:10.1186/1745-6215-13-43

Cite this article as: Jäger et al:: Effects of a manualized short-term

treatment of internet and computer game addiction (STICA): study protocol for a randomized controlled trial. Trials 2012 13:43.

\section{Submit your next manuscript to BioMed Central} and take full advantage of:

- Convenient online submission

- Thorough peer review

- No space constraints or color figure charges

- Immediate publication on acceptance

- Inclusion in PubMed, CAS, Scopus and Google Scholar

- Research which is freely available for redistribution 